\title{
Origin of Low Detection Limit and High Selectivity of Roche Accu-Chek Test Strips that Enables Measurement of Tear Glucose Levels
}

\author{
Kyoung Ha Cha, ${ }^{[a]}$ Yu Qin, ${ }^{[a]}$ and Mark E. Meyerhoff*[a]
}

\begin{abstract}
Tear glucose measurements have been suggested as a potential alternative to blood glucose monitoring for diabetic patients. While previous work has reported a correlation between blood and tear glucose levels in humans, this link has not been thoroughly established and additional clinical studies are needed. We recently reported that Roche's electrochemical Accu-Chek blood glucose test strips exhibit far superior analytical performance over other commercial brands, with the low detection
\end{abstract}

limit and high selectivity required for quantitating tear glucose levels. Herein, we evaluate the origin of the high sensitivity and selectivity of the Roche test strips and show that the use of pyrroloquinoline quinone (PQQ)-dependent glucose dehydrogenase (GDH) as an active enzyme reagent in combination with a nitrosoaniline derivative as an electron transfer mediator are primarily responsible for the low limit of quantification $(L O Q$ ) (ca. $9 \mu \mathrm{M}$ ) and enhanced selectivity achieved with these strips.

Keywords: Tear glucose • Roche Accu-Chek glucometer strips • Biamperometry - PQQ-dependent glucose dehydrogenase • Nitrosoaniline derivative

\section{Introduction}

The World Health Organization reports that 347 million people worldwide have diabetes [1] and projects that diabetes will be the $7^{\text {th }}$ leading cause of death in 2030 [2], despite the advancing modern medical science and technology $[3,4]$. Tight glycemic control is essential to prevent or reduce life-threatening hyper- and hypoglycemic crises causing serious long-term complications [5]. Using a personal blood glucometer with an enzymatically-active disposable test strip, enables diabetic patients to monitor their glucose levels by obtaining a small drop of capillary blood from their fingertips or forearms with a conventional lancet. While minimum of four measurements of glucose per day is recommended to maintain normal levels of glucose in blood [6], up to eight checks per day are recommended for children and adolescents with Type 1 diabetes on insulin treatment. ${ }^{7}$ Pricking the finger tip for many times a day can limit patient compliance and develop massive scarring/callous formation and loss of sensibility/perception hindrance. Consequently, it may lead to suboptimal blood glucose control and induce hypoglycemia. Therefore, the development and availability of a pain-free glucose measuring device could greatly increase patient compliance and maintain optimal glucose control.

Tear glucose measurements have been suggested as an alternative method to monitor glucose level. Tear glucose measurement techniques mandate a low micromolar $L O Q$, high selectivity over potential interferences such as ascorbic acid, uric acid and acetaminophen, and measurement within microliter or sub-microliter sample volume. Since several studies have shown that there may be a clini- cally useful correlation between blood and tear glucose levels [7-9], more detailed studies are necessary to confirm the potential utility of tear glucose measurements to help maintain optimal glucose control. We recently reported [10] that Roche's Accu-Chek blood glucose test strips are able to measure glucose levels in tear samples with adequate sensitivity $(0.127 \mathrm{nA} / \mu \mathrm{M})$ and selectivity with $\leq 13 \%$ error $(n=3)$ for 25,50 and $75 \mu \mathrm{M}$ glucose in the presence of $10 \mu \mathrm{M}$ acetaminophen, $100 \mu \mathrm{M}$ ascorbic acid, and $100 \mu \mathrm{M}$ uric acid. In this earlier work, the Roche test strips were used for measurement of glucose concentration in tears from nine normal (non-diabetic) fasting human subjects and reported glucose values were within the range of $5-148 \mu \mathrm{M}$ (mean $=47 \mu \mathrm{M}$, median $=$ $43 \mu \mathrm{M})$, a similar range to that reported by Asher and coworkers using an LC-MS technique. ${ }^{8}$ Herein, we utilize bulk phase solution experiments with different enzymes, strip-based screen printed electrode materials and mediators to explain why the Roche test strips exhibit such excellent detection limits and selectivity compared to all other strips examined in our earlier studies [10]. Results indicate that the unique combination of pyrroloquinoline quinone dependent glucose dehydrogenase (PQQ-GDH) as the active enzyme and a nitrosoaniline derivative as the electron transfer mediator are the key components of this test strip that yield the required sensitivity and selectivity to accurately measure low glucose levels in tear fluid.

[a] K. H. Cha, Y. Qin, M. E. Meyerhoff Department of Chemistry, University of Michigan Ann Arbor, MI, 48109-1055, USA

*e-mail: mmeyerho@umich.edu 


\section{Experimental}

\subsection{Chemicals and Reagents}

D-(+)-Glucose, glucose oxidase from A. niger (149.8 U/ $\mathrm{mg})$, sodium chloride $(\mathrm{NaCl})$, potassium ferricyanide(III) $\left(\mathrm{K}_{3} \mathrm{Fe}(\mathrm{CN})_{6}\right)$, potassium chloride $(\mathrm{KCl})$, sodium phosphate dibasic $\left(\mathrm{Na}_{2} \mathrm{HPO}_{4}\right)$, potassium phosphate monobasic $\left(\mathrm{KH}_{2} \mathrm{PO}_{4}\right)$, L-ascorbic acid, uric acid sodium salt, and acetaminophen were purchased from Sigma-Aldrich (St. Louis, MO). FAD (flavin adenine dinucleotide)-dependent glucose dehydrogenase from Aspergillus sp. (1180 U/ $\mathrm{mg}$ ) was obtained from Sekisui Enzymes (Lexington, MA). PQQ-dependent glucose dehydrogenase (808 U/ $\mathrm{mg}$ ) was a product of Toyobo Enzyme (Osaka, Japan). $O$ methoxy-[N,N-bis-(2-hydroxyethyl)]- $p$-nitrosoaniline (nitrosoaniline derivative) (see Figure 1) was generously donated by PharmaBlock R\&D Co. Ltd (Nanjing, China).

\subsection{Chemicals and Reagents}

A four-channel Biostat (ESA Biosciences Inc., Chelmsford, MA) was used to measure the amperometric response to glucose solutions $(0-5 \mathrm{mM})$ containing enzymes, mediators and/or potential interfering species (easily oxidizable species at applied potentials) using an applied potential of $+150 \mathrm{mV}$. For testing electrodes with different materials (palladium, gold and carbon), commercially available glucose test strips from Bayer, Roche Diagnostics and Abbott were purchased over the counter and the top housing layers were removed to expose the electrochemical sensing sites. Each of these strips uses palladium, gold and carbon as the working electrode material, respectively. The exposed surfaces of the strips were then washed thoroughly with deionized water and ethanol to remove any deposited chemical reagents on the surface of electrodes before use in bulk solution experiments.

Individual stock solutions of $1.5 \mathrm{M}$ glucose, $25 \mathrm{mM}$ ascorbic acid, $25 \mathrm{mM}$ uric acid and $25 \mathrm{mM}$ of acetaminophen were made in phosphate saline buffer (PBS) solution containing $137 \mathrm{mM} \mathrm{NaCl}, 2.7 \mathrm{mM} \mathrm{KCl}, 10 \mathrm{mM}$ $\mathrm{Na}_{2} \mathrm{HPO}_{4}$, and $2 \mathrm{mM} \mathrm{K \textrm {K } _ { 2 }} \mathrm{PO}_{4}$, adjusted $\mathrm{pH}$ to 7.4 with $1 \mathrm{M} \mathrm{NaOH}$. Standard solutions were prepared by dilution of the stock solutions using PBS solution for calibrations of glucose. Solutions of $0.5 \mathrm{mM}$ ascorbic and uric acid and $0.2 \mathrm{mM}$ acetaminophen (freshly made each day) were used to examine the cumulative effect of these potential interference species on the accuracy of glucose measurements. The reported average levels of ascorbic and uric acid in tears are $20 \pm 6.2$ and $68 \pm 46 \mu \mathrm{M}$, respectively $[11,12]$. A $0.2 \mathrm{mM}$ acetaminophen solution was also tested assuming that this species may be present in tears at a relative dilution ratio similar to blood glucose, if the patient was using this drug. Stock solutions of the various enzymes and mediators were made in PBS solution in the same way.

\subsection{Electrochemical Measurements}

Various glucometer strips for which the reagents layers were removed and the electrodes first exposed and cleaned were used as biamperometric detectors in bulk solution phase experiments. Each of the strips has two identical electrodes that are utilized for glucose detection with one electrode employed as the working electrode (apply positive voltage to oxidize reduced form of mediator formed from enzymatic oxidation of glucose and/or reduction of mediator by potential interferent species that can directly reduce the mediator) and the other serving as the pseudo reference/counter electrode. The cleaned and uncovered electrode ends of the various strips were placed in $5 \mathrm{~mL}$ of PBS buffer within a vial, and a small magnetic stir bar was utilized to mix the solution. Different mediators (e.g., ferricyanide or the nitrosoaniline derivative (see Figure 1)) alone or in combination with different glucose oxidizing enzymes (at near equivalent activity in term of Units $/ \mathrm{mL}$ ) were added to the PBS solution for testing response to ascorbic acid, uric acid and acetaminophen, as well as to different concentrations of glucose. The Biostat electrochemical analyzer was used to apply the indicated voltage between the two electrodes of the strips and to monitor the resulting current as a function of time after addition of glucose or test interference species.

\section{Results and Discussion}

A schematic of a generic blood glucometer test strip when used for monitoring glucose in tear fluid is illustrat-

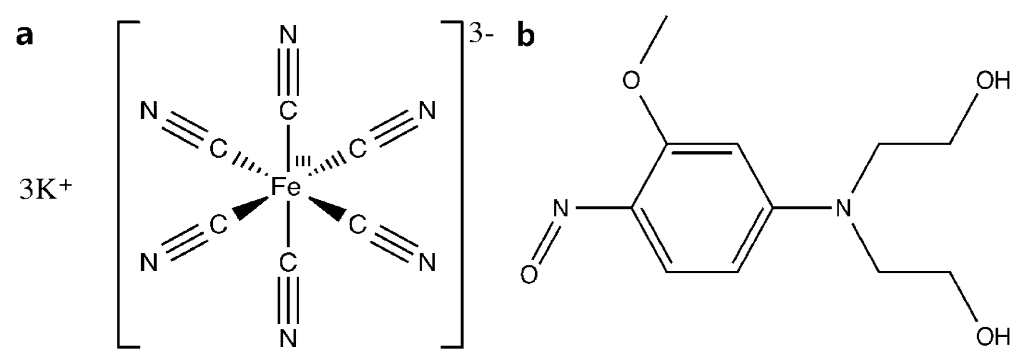

Fig. 1. Structures of (a) ferricyanide and (b) $O$-methoxy-[N,N-bis-(2-hydroxyethyl)]-p-nitrosoaniline that were examined as electron transfer mediators in this work. 


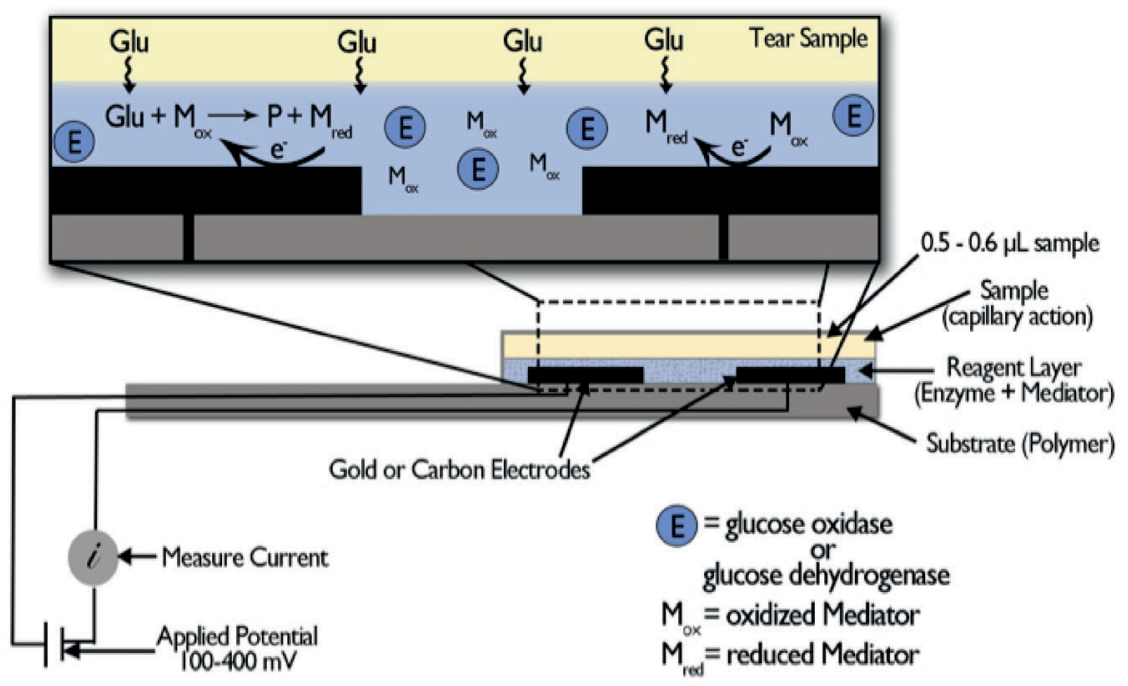

Fig. 2. Schematic of blood glucometer test strip and its potential application for monitoring glucose levels in a tiny volume of tear fluid. In addition to gold and carbon electrodes utilized in a bioamperometric sensing mode, Pd and other metals have been employed.

ed in Figure 2. A number of different glucose-oxidizing enzymes have been used to prepare conventional glucose biosensors for measuring glucose concentration in whole blood. Glucose oxidase (GOX) has been widely used to catalyze oxidation of glucose on or close to the electrode surface, which can be followed by amperometric monitoring of liberated $\mathrm{H}_{2} \mathrm{O}_{2}$ or the consumed $\mathrm{O}_{2}$. However, monitoring peroxide production when using GOX requires a high applied potential $(+600 \mathrm{mV})$ [13], at which potential interferents can also be easily oxidized. Fortunately, via the aid of electron transfer mediators the applied potential required can be significantly lowered to partially reduce the interference problem. Indeed, efficient electron transfer mediators such as ferrocene and its derivatives were originally used for the enzymatic reaction catalyzed by GOX and applied in Abbott's Medisense Precision Q.I.D. blood glucometer test strips back in the early 1990s. However, since then, other mediators including potassium ferricyanide, osmium complexes and nitrosoaniline derivatives have become the mediators of choice and are the ones most widely used in current commercial blood glucometer test strips. In fact, several commercial strips (e.g., Johnson and Johnson One Touch, Bayer Contour, etc.) utilize potassium ferricyanide as the mediator in the strip configuration illustrated in Figure 2. Beyond glucose oxidase, two different types of glucose dehydrogenase $(\mathrm{GDH})$ enzymes are also often employed to create blood glucometer strips; FAD dependent GDH, and PQQ dependent GDH. The Roche test strips that were found to be so useful for tear glucose measurements utilize a unique combination of gold working/counter electrodes, PQQ-GDH as an active enzyme and a nitrosoaniline derivative (see Figure 1) as an electron transfer mediator. This strip is able to measure low concentrations glucose found in tears selectively in the presence of electroactive interferents [10]. An optimal applied voltage of $150 \mathrm{mV}$ between the two gold electrodes within the Roche strips for calibration over the 1$100 \mu \mathrm{M}$ glucose concentration range was found to yield the lowest \% errors from such potential interferent species [10].

To better understand the enhanced selectivity over potential interferences of the Roche Accu-Chek glucometer strips vs. other common brands when applied for tear measurements, we first examined the response of interfering species (ascorbic acid, uric acid and acetaminophen) on three different electrode strip materials (palladium, gold and carbon) in terms of the steady-state amperometric responses in the presence of two different electron transfer mediators, potassium ferricyanide and the nitrosoaniline derivative (Figure 1), in bulk solution experiments. As stated above, potassium ferricyanide is the most widely used electron transfer mediator at present and therefore it was chosen to be compared directly to the nitrosoaniline derivative. Our aim was to determine which combination yields the least current from oxidation of the potential interferents. An applied potential of $+150 \mathrm{mV}$ between the two strip electrodes was chosen because it is the optimal potential found previously to achieve the highest selectivity for glucose for the Roche test strips with nitrosoaniline derivative as an electron transfer mediator [10]. Further, this applied potential is close to the $+200 \mathrm{mV}$ value that is generally used for test strips employing potassium ferricyanide as an electron transfer mediator [14].

As shown in Figure 3, on gold-printed electrodes, a large increase in current is observed at the $80 \mathrm{~s}$ mark when ascorbic acid is added to a stirred PBS solution ( $\mathrm{pH} 7.4$ ) to achieve a final concentration of $0.5 \mathrm{mM}$ ascorbic acid for sensor strips with either potassium ferricyanide or nitrosoaniline derivative as the added redox mediators in the bulk solution and with $+150 \mathrm{mV}$ applied be- 

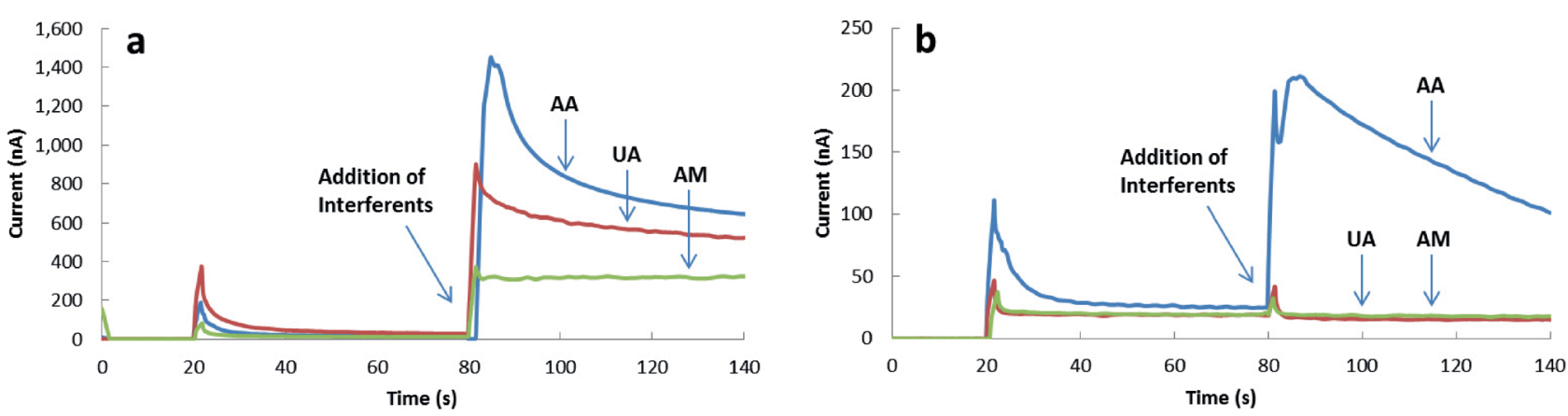

Fig. 3. Comparison of dynamic amperometric responses (at room temperature; applied potential $=+150 \mathrm{mV}$ ) from the interferents (0.5 mM ascorbic acid (AA), $0.5 \mathrm{mM}$ uric acid (UA) and $0.2 \mathrm{mM}$ acetaminophen (AM)) in the presence of $10 \mathrm{mM}$ potassium ferricyanide (a) or $10 \mathrm{mM}$ nitrosoaniline derivative (b) in PBS ( $\mathrm{pH}$ 7.4, $0 \mu \mathrm{M}$ glucose) on gold-printed electrodes. Background response from $10 \mathrm{mM}$ potassium ferricyanide (a) and nitrosoanaline derivative in PBS solution is recorded between 20-80 s. Response from interferents are recorded between $80-140 \mathrm{~s}$.

tween working and reference/counter electrode of the strips. It should also be noted that the level of currents observed toward ascorbic acid is much greater when ferricyanide is used vs. when an equivalent concentration of nitrosoanaline deriative is employed in the bulk solution $(10 \mathrm{mM})$ under the exact same experimental conditions. However, when nitrosoaniline derivative is employed (Figure $3 \mathrm{~b}$ ), $0.5 \mathrm{mM}$ ascorbic acid is the only potential interferent species yielding a significant current at $+150 \mathrm{mV}$ applied potential (primarily due to reduction of the mediator by ascorbate). As shown in Figure $3 \mathrm{~b}$, $0.5 \mathrm{mM}$ uric acid and $0.2 \mathrm{mM}$ acetaminophen are not electro-oxidized at all when the nitroaniline derivative is used at $10 \mathrm{mM}$ levels in the PBS solution. In contrast, when potassium ferricyanide is employed as mediator, relatively large responses from uric acid and acetaminophen are observed at an applied potential of $+150 \mathrm{mV}$ (Figure $3 \mathrm{a}$ ). This same behavior is also observed for palladium and carbon-printed electrodes (data not shown). When using potassium ferricyanide as mediator, all three types of electrodes (palladium, gold and carbon) exhibit a significant current increase in the presence of potential interferents, while in the presense of the nitrosoaniline derivative, $0.5 \mathrm{mM}$ uric acid and $0.2 \mathrm{mM}$ acetaminophen do not yield an increase in anodic current. It is well known that, ascorbic acid can react with the mediators in their oxidized form to form reduced mediator which can then be oxidized electrochemically. Our results indicate that the nitrosoaniline derivative is much more inert to these interfering species than potassium ferricyanide, which makes the nitrosoaniline derivative a better electron transfer mediator for more selective glucose measurements in blood as well as in tear fluid.

Initial screening of all possible combinations of different electrode materials and mediators indicated that the use of gold working/counter electrodes along with the nitrosoanaline derivative is the only combination that exhibits the optimal selsectivity over potential interferents in the bulk phase experiments using the printed electrodes of several commercial brands of test strips. Consequently, this combination was utilized in further studies to measure low-end glucose concentrations using different glucose oxidizing enzymes as catalysts for detection of glucose.

Toward this end, three different enzymes used for glucose measurements were examined in the bulk phase experiments using biamperometric detection with various glucometer strip electrodes. The enzymes tested included glucose oxidase, as well as glucose dehydrogenases with two different cofactors/prosthetic groups, flavin adenine dinucleotide (FAD) and pyrroloquinoline quinone (PQQ). The enzymes were added to the PBS buffer alone with either potassium ferricyanide or nitrosoaniline derivative, and the biamperometric response toward additions of glucose concentrations in the range of $0-5 \mathrm{mM}$ were recorded. The response curves toward glucose were obtained initially in PBS without any interfering species present. As shown in Figure $4 \mathrm{a}$, with $125 \mathrm{U} / \mathrm{mL}$ FAD- or PQQ-dependent GDHs, coupled with $20 \mathrm{mM}$ potassium ferricyanide in the test solution and gold electrodes as the biamperometric detectors (at an applied potential of $+150 \mathrm{mV}$ ), significant amperometric signals are observed for low-end glucose concentrations in the range of 0-0.2 mM. In contrast, when using GOX at the same $\mathrm{U} / \mathrm{mL}$ concentrations, there is very poor sensitivity to changes in glucose concentrations, with amperometric response observed only in the higher concentrations range $(\geq 0.5 \mathrm{mM})$. In the case of the nitrosoaniline derivative (Figure 4b), only the PQQ-GDH catalyst yields significant amperometric response to glucose in the low concentration range required for tear glucose measurements. It is important to note that $20 \mathrm{mM}$ of both mediators was used for these experiments, instead of $10 \mathrm{mM}$, in order to have a sufficient level of mediator necessary to oxidize high-end glucose concentrations.

The far superior glucose sensitivity observed in the above experiments when using both FAD- and PQQ-dependent GDH may relate to their lower MichaelisMenten constant $K_{\mathrm{M}}$ values, compared to the other enzymes that catalyze glucose oxidation. The lower $K_{\mathrm{M}}$ results in an increase in the rate of reaction at a lower glucose level and, therefore, increased catalytic current from 


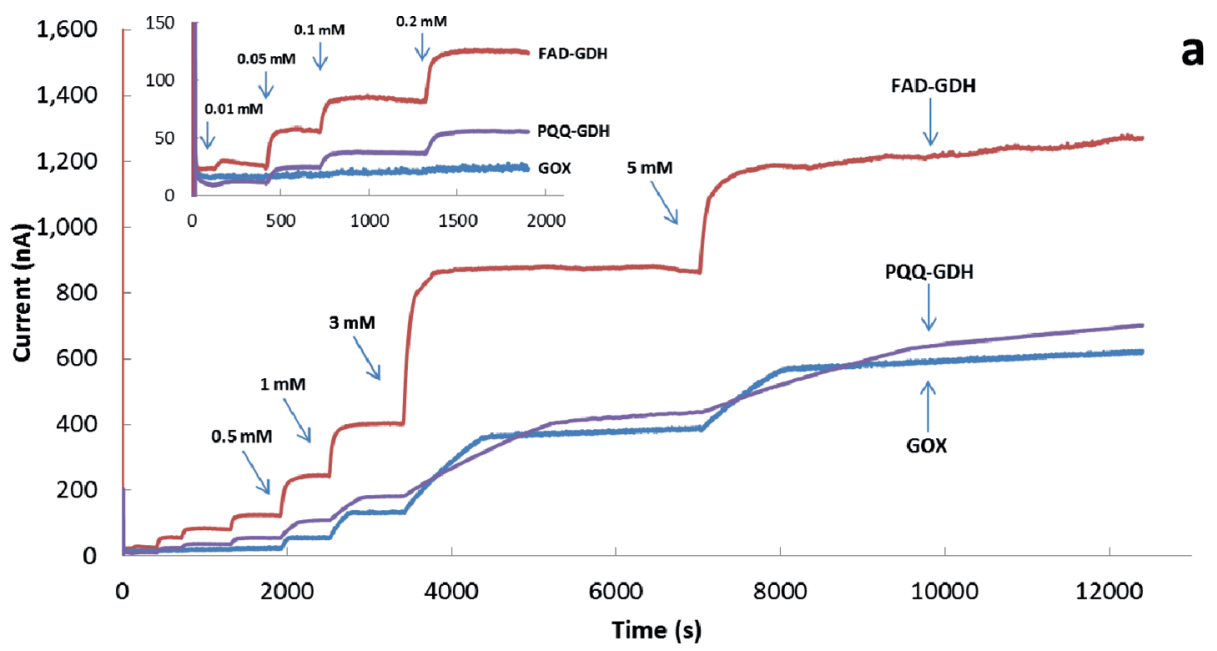

a

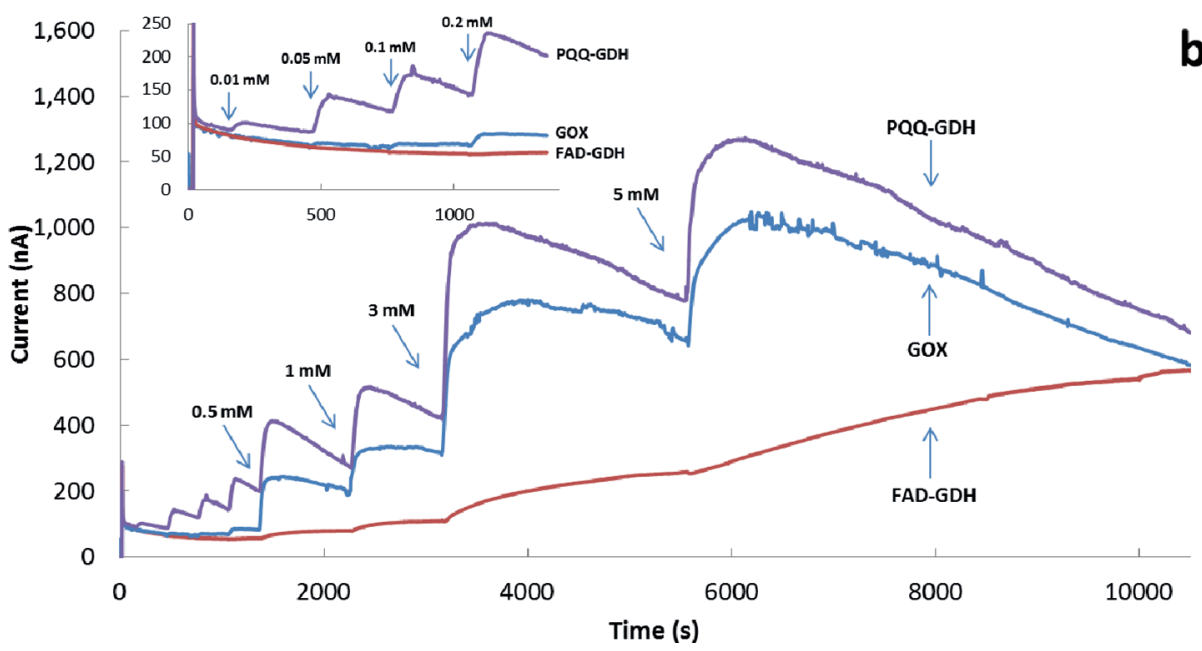

Fig. 4. Dynamic amperometric response (at room temperature; applied potential $=+150 \mathrm{mV}$ ) for changes in glucose concentration $(0-5 \mathrm{mM})$ in the presence of (a) $20 \mathrm{mM}$ potassium ferricyanide and (b) $20 \mathrm{mM}$ nitrosoaniline derivative in PBS solution (pH 7.4) with $125 \mathrm{U} / \mathrm{ml}$ glucose oxidase (GOX), FAD- (FAD-GDH), and PQQ-dependent glucose dehydrogenase (PQQ-GDH). The activities of the enzymes were $149.8 \mathrm{U} / \mathrm{mg}, 1180 \mathrm{U} / \mathrm{mg}$ and $808 \mathrm{U} / \mathrm{mg}$, respectively. Insets: Current response from low-end glucose concentrations $(0-0.2 \mathrm{mM})$. Within the figure, the indicated concentrations represent the total concentration of glucose at each arrow point in time.

oxidation of the reduced mediator at the working electrode at lower glucose concentrations. Indeed the $\mathrm{Mi}$ chaelis-Menten constant $K_{\mathrm{M}}$ values toward glucose of GOX, FAD-GDH, NAD-GDH, and PQQ-GDH are reported to be $27,0.46,10$ and $0.38 \mathrm{mM}$, respectively [15$18]$. Hence, there is a significantly lower $K_{\mathrm{M}}$ value for the two enzymes that exhibit the most sensitive response to glucose levels in the bulk solution phase experiments. Further, from the patent literature [19], it is known that Roche utilizes a recombinant form of PQQ-GDH in order to avoid interference from maltose that can cause errors with blood glucometer devices that utilize PQQ$\mathrm{GDH}$ as the active enzyme. Hence, it is possible that the recombinant form of PQQ-GDH utilized in the AccuChek strips may have an even lower $K_{\mathrm{M}}$ compared to the $0.38 \mathrm{mM}$ value reported for the native enzyme.

Although use of FAD- and PQQ-dependent GDHs in combination with potassium ferricyanide as the electron transfer mediator exhibits very good sensitivity for low concentration detection of glucose, the use of potassium ferricyanide is not desired since a considerable amount of current is detected from oxidation of ascorbic acid, uric acid and acetaminophen using this mediator (see Figure $3 \mathrm{a}$, above). Indeed, when potassium ferricyanide is employed as the electron transfer mediator, the lower detection limit for the bulk phase experiments is only ca. $0.5 \mathrm{mM}$ glucose, when $0.5 \mathrm{mM}$ ascorbic acid, $0.5 \mathrm{mM}$ uric acid and $0.2 \mathrm{mM}$ acetaminophen are all present in the test solution (data not shown).

As stated above, when using nitrosoaniline derivative as the electron transfer mediator (Figure 4b), PQQ-GDH produces the most sensitive response to glucose among all three enzymes tested, especially in the desired tear glucose measuring range $(0-0.2 \mathrm{mM})$. With the nitrosoaniline derivative, GOX and FAD-GDH only yield amperometric response to glucose in the higher range of glucose levels $(\geq 0.5 \mathrm{mM})$. It should be noted that for same enzyme, the sensitivity differs significantly with the differ- 

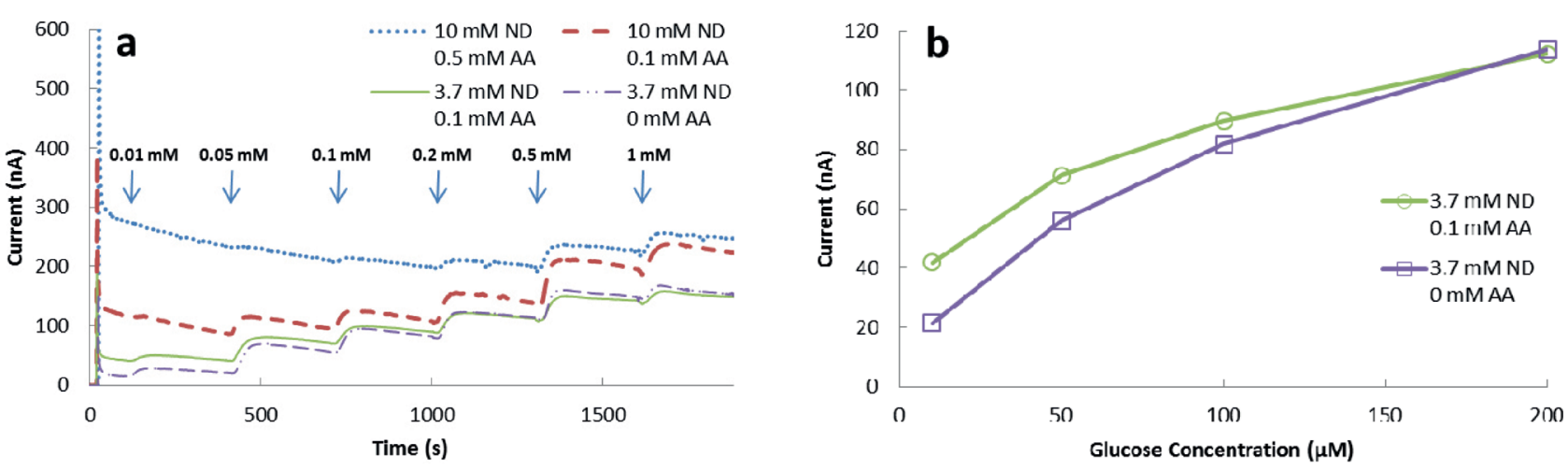

Fig. 5. (a) Dynamic amperometric response (at room temperature; applied potential $=+150 \mathrm{mV}$ ) for increasing glucose concentrations $(0-1 \mathrm{mM})$ in the presence of 10 and $3.7 \mathrm{mM}$ nitrosoaniline derivative (ND), PQQ-dependent glucose dehydrogenase with various concentration of ascorbic acid (AA; 0, 0.1 and $0.5 \mathrm{mM}$ ) in PBS solution (pH 7.4). Within the figure, concentrations refer to the total concentration of glucose made at each arrow point in time. (b) Dependence of steaedy-state current on glucose concentration in the presence of $0.1 \mathrm{mM}$ ascorbic acid (circles) and without ascorbic acid (squares).

ent mediators. Our results indicate that for FAD-GDH, potassium ferricyanide is a better mediator than nitrosoaniline for facilitating the electron-transfer process. However, for PQQ-GDH, the use of the nitrosoaniline derivative is required to obtain enhanced sensitivity, and the selectivity over interfering species is far superior to the combination of FAD-GDH and potassium ferricyanide. Another advantage of mediator facilitated PQQ-GDH sensing system is its oxygen independence. We compared glucose calibrations $(0-3 \mathrm{mM})$ in bulk solution containing PQQ-GDH and nitrosoaniline derivative in the presence of ambient oxygen concentration $(21 \%)$ and with no oxygen present by purging with $\mathrm{N}_{2}$. The sensitivity and low detection limit in both experiments are nearly identical, suggesting that for amperometric glucose measurements, use of the combination of PQQ-GDH and the nitrosoaniline derivative yields signals that are independent of oxygen levels in the solution (data not shown).

The electrochemical response of the strips made with gold electrodes to glucose in the presence of ascorbic acid in the bulk solution was also examined using the optimal combination of PQQ-GDH and the nitrosoaniline derivative. The previous results (see Figure 3 ) suggested that ascorbic acid still poses a problem and that the combination of PQQ-GDH, nitrosoaniline derivative and gold electrode detection cannot avoid oxidation of ascorbic acid at an applied potential of $+150 \mathrm{mV}$. As shown in Figure $5 \mathrm{a}$, with $10 \mathrm{mM}$ nitrosoaniline derivative present in solution, the glucose sensitivity at low concentrations is better in the presence of $0.1 \mathrm{mM}$ ascorbic acid than $0.5 \mathrm{mM}$ ascorbic acid. However, as also shown in Figure $5 \mathrm{a}$, the interference effect from ascorbic acid can be partially minimized by controlling the concentration of the mediator, through which the reaction between a lower concentration of nitrosoaniline derivative $(3.7 \mathrm{mM})$ and $0.1 \mathrm{mM}$ ascorbic acid yields less anodic current than the reaction between $10 \mathrm{mM}$ nitrosoaniline derivative and $0.1 \mathrm{mM}$ ascorbic acid. Figure $5 \mathrm{~b}$ shows the glucose calibration curves for the gold-printed electrodes coupled with PQQ-
GDH and $3.7 \mathrm{mM}$ nitrosoaniline derivative (in bulk solution) in the absence and presence of $0.1 \mathrm{mM}$ ascorbic acid. Although in the range of $0-50 \mu \mathrm{M}$ glucose, the oxidation current from ascorbic acid is high, at the $50 \mu \mathrm{M}$ glucose level, the current increase by the presence of $0.1 \mathrm{mM}$ ascorbic acid is ca. $27 \%$, corresponding to $+13 \mu \mathrm{M}$ error in the glucose measurement. At $200 \mu \mathrm{M}$ glucose, the error from ascorbic acid is only $1 \%$. However, in the prior work with fully assembled Roche AccuChek strips [10], we found much lower errors when ascorbate at $0.1 \mathrm{mM}$ in combination with acetaminophen and uric acid was tested as an interference solution in the presence of $50 \mu \mathrm{M}$ glucose (combined error from interferences $<13 \%$ [10]). Since in the fully assembled commercial blood glucometer strip, the PQQ-GDH and nitrosoaniline derivative ( $1: 2$ by weight) are coated over the surface of the gold electrodes within a polymeric matrix containing thickeners, stabilizers, detergents, and other adjunct materials, and the current is recorded after $5 \mathrm{~s}$ after wicking of the test samples into the strips, there may well be some partial mass transport rejection of ascorbic acid into the enzyme/mediator layer that further enhances selectivity compared to the homogeneous experiments reported in this work.

\section{Conclusion}

In this report, we have shown that that the ability of Roche Accu-Chek test strips to be used to measure $\mu \mathrm{M}$ levels of glucose without substantial interference from other redox active species is due to the unique combination of using PQQ-GDH as the active enzyme in combination with nitrosoaniline derivative as an electron transfer mediator. The low $K_{\mathrm{M}}$ value of this enzyme, coupled with the inability of uric acid and acetaminophen to reduce the nitrosoaniline mediator and yield electrochemical response in a biamperometric measurement mode, appear to be the key elements that provide the excellent $L O Q$ value and selectivity of these strips. These findings 


\section{Full Paper}

suggest that any future efforts to create specialized electrochemical glucometer type test strips, specifically for direct sampling of submicroliter quantities of tear fluid from eyes, should utilize PQQ-GDH in combination with O-methoxy-[N,N-bis-(2-hydroxyethyl)]-p-nitrosoaniline as the reagents.

\section{Acknowledgements}

We gratefully thank EyeLab LLC (Ann Arbor, MI) for supporting these studies.

\section{References}

[1] G. Danaei, M. M. Finucane, Y. Lu, G. M. Singh, M. J. Cowan, C. J. Paciorek, J. K. Lin, F. Farzadfar, Y. H. Khang, G. A. Stevens, M. Rao, M. K. Ali, L. M. Riley, C. A. Robinson, M. Ezzati, Lancet 2011, 378, 31-40.

[2] Global Status Report on Noncommunicable Diseases 2010, World Health Organization, Geneva, 2011.

[3] P. Zimmet, K. G. M. M. Alberti, J. Shaw, Nature 2001, 414, $782-787$.

[4] M. Brownlee, Nature 2001, 414, 813-820.

[5] J. Hönes, P. Müller, N. Surridge, Diabetes Technol. Ther. 2008, 10, S10-S26.

[6] E. Minder, D. Albrecht, J. Schäfer, H. Zulewski, Diabetes Res. Clin. Pract. 2013, 101, 57-61.
[7] J. K. Lane, D. M. Krumholz, R. A. Sack, C. Morris, Curr Eye Res. 2006, 31, 895-901.

[8] J. T. Baca, C. R. Taormina, E. Feingold, D. N. Finegold, J. J Grabowski, S. A. Asher, Clin. Chem. 2007, 53, 1370-1372

[9] C. R. Taormina, J. T. Baca, S. A. Asher, J. J. Grabowski, J. Am. Soc. Mass. Spec. 2007, 18, 332-336.

[10] K. H. Cha, G. C. Jensen, A. S. Balijepalli, B. E. Cohan, M. E. Meyerhoff, Anal. Chem. 2014, 86, 1902-1908.

[11] C. K. M. Choy, I. F. F. Benzie, P. Cho, Invest. Ophthalmol. Vis. Sci. 2000, 41, 3293-3298.

[12] C. K. M. Choy, P. Cho, W. Y. Chung, I. F. F. Benzie, Optom. Vis. Sci. 2003, 80, 632-636.

[13] H. Yamaoka, K. Sode, Open Biotechnol. J. 2007, 1, 26-30.

[14] Z. P. Tang, R. F. Louie, J. H. Lee, D. M. Lee, E. E. Miller, G. Kost, J. Crit. Care Med. 2001, 29, 1062-1070.

[15] R. Wilson, A. P. F. Turner, Biosens. Bioelectron. 1992, 7, $165-185$.

[16] D. Fapyane, S. J. Lee, S. H. Kang, D. H. Lim, K. K. Cho, T. H. Nam, J. P. Ahn, J. H. Ahn, S. W. Kim, I. S. Chang, Phys. Chem. Chem. Phys. 2013, 15, 9508-9512.

[17] L. D. Smith, N. Budgen, S. J. Bungard, M. J. Danson, D. W. Hough, Biochem. J. 1989, 261, 973-977.

[18] X. Yuan, M. Iijima, M. Oishi, Y. Nagasaki, Langmuir, 2008, 24, 6903-6909.

[19] D. W. Burke, M. Marquant, Test Strip with Slot Vent Opening. U. S. Patent 8,211, 379, July 3, 2012.

Received: October 13, 2014

Accepted: November 11, 2014 Published online: February 2, 2015 